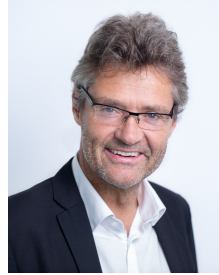

\section{Jörg Freiling}

Lehrstuhl für Mittelstand, Existenzgründung und Entrepreneurship (LEMEX), Universität Bremen

freiling@uni-bremen.de

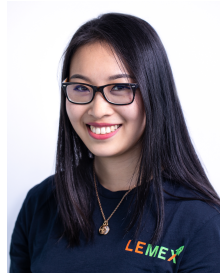

\section{Quynh Duong Phuong}

Projektmitarbeiterin \& Innovation Broker (LEMEX), Universität Bremen

phuong@uni-bremen.de

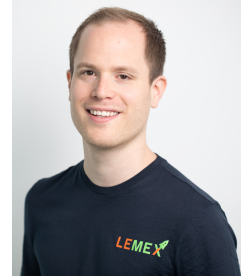

\section{Thomas Baron}

Postdoc, Projektmitarbeiter \& Innovation Broker (LEMEX), Universität Bremen

thomas.baron@uni-bremen.de

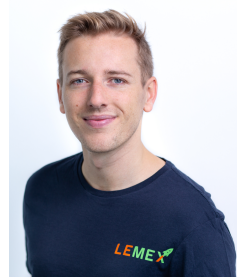

Jan Elsner

Projektmitarbeiter \& Innovation Broker (LEMEX), Universität Bremen

jan.elsner@uni-bremen.de

\title{
Inno-Quarters - mit Living Labs auf Festivals zur Validierung neuer Geschäftsmodelle
}

Wenn der Hauptgrund des Scheiterns neuer Geschäftskonzepte die fehlende Marktnähe ist, sind innovative Instrumente zur Einholung frühen Markt-Feedbacks und zum Co-Development mit Zielkunden höchst willkommen. Inno-Quarters sind solche Bühnen, die Entrepreneure und Feedback gebende Zielkunden in entspannter, kreativer Atmosphäre - hier: im Rahmen von Festivals - in den gemeinsamen Austausch bringen. Da Feedback aber fehlinterpretiert werden kann und auch nicht immer aus der Kernzielgruppe stammt, müssen Inno-Quarters sorgfältig vorbereitet werden. Dieser Beitrag zeigt, welche organisatorischen Grundlagen für die Schaffung solcher Living Labs geschaffen werden müssen und was zu tun ist, um Entrepreneure auf das Event vorzubereiten und das Feedback im Sinne einer zielführenden Geschäftsentwicklung auszuwerten. Die Ausführungen stützen sich auf die Erfahrung bei der Ausrichtung zahlreicher Inno-Quarters in Europa. 


\section{Einleitung}

Viele neue Geschäftsmodelle scheitern oder müssen grundsätzlichen Änderungen unterzogen werden, weil die Marktfähigkeit verlorengegangen ist oder nie bestand. Konzepte wie Design Thinking, Customer Development oder Lean Startup ${ }^{1}$ greifen diese Problematik auf und betonen die Relevanz von frühphasigem Nutzerfeedback zur Validierung der Markteignung neuer Geschäftsmodelle. Allerdings stehen viele Entrepreneure vor der Herausforderung, diesen interaktiven Ansatz in der Praxis konkret umzusetzen.

\section{Was sind Living Labs und „Inno-Quarters“?}

Living Labs sind nutzerzentrierte Formate für Open Innovation Aktivitäten, in denen Innovatoren Anwender zum Zwecke des Co-Developments von Lösungen ebenso einbeziehen wie andere nützliche Stakeholder (z.B. Hochschulen, Branchenexperten, öffentliche Hand). Es entsteht so eine Innovations-Community, die entlang der Lebensrealität auf experimentellem Wege nutzergerechte Lösungen schafft.

Inno-Quarters sind Bühnen zur systematischen und begleiteten Einholung von Feedback zwecks Entwicklung neuer Geschäftskonzeptionen durch Startup oder Corporate Entrepreneure. Die Entrepreneure werden in professioneller Feedback-Einholung geschult und bei der Auswertung zur Weiterentwicklung der Geschäftskonzeption begleitet. Inno-Quarters werden als Teil von Veranstaltungen wie Festivals eingerichtet und zielen auf die offene Kommunikation zwischen Entrepreneuren und Teilnehmenden der Events.
Was der Entwicklung zusätzlichen Schwung verleiht, ist die Implementierung von Explorationsformaten, die systematisches Feedback von potenziellen Nutzern einfangen und umzusetzen helfen. Hierzu dienen sog. "Inno-Quarters", die auf bestehenden Veranstaltungen eingerichtet werden, um Startup- (oder auch Corporate) Entrepreneuren eine Bühne zur Einholung offenen Feedbacks zu ihrer Geschäftskonzeption zu bieten. Was sich auf den ersten Blick recht simpel anhören mag, um zum entsprechenden Ergebnis zu führen, ist in der konkreten Umsetzung jedoch an Voraussetzungen und vor allem an eine gründliche Vorbereitung gebunden.

Dieser Beitrag stellt auf das von der EU im Rahmen des Interreg-Programms geförderte „Inno-Quarter“-Projekt ab, das sich derartigen Living Labs auf Festivals

annimmt ${ }^{2}$. Er spezifiziert das Grundproblem, behandelt die Entstehungsgeschichte, Inhalte und Grundlagen von Inno-Quarters und fokussiert auf die Realisierungsschritte, Begleitstrukturen und Faktoren zur erfolgreichen Umsetzung auf Basis praktischer Erfahrungen für potenzielle Organisatoren solcher Inno-Quarters, sei es im CorporateKontext oder für Manager von Innovationsförderprogrammen.

\section{Problemstellung und Vorgehensweise}

Neugeschäftsinitiativen, insbesondere von Startups, stehen von Beginn an vor der Frage der Marktrelevanz des identifizierten Kundenproblems und der Marktfähigkeit der 
Lösung. Studien zum Scheitern von Jungbetrieben sind sich einig, dass Faktoren im Kontext des sog. „Product/Market Fits“ (siehe Box) die größte Hürde darstellen. Am Markt vorbeigedacht und -produziert zu haben, ist demzufolge der häufigste Abbruchgrund der Entwicklung neuer Geschäftskonzeptionen³ .

Umso verständlicher ist es, dass im Kontext neuer Ansätze des Gründens sowie des agilen Arbeitens zahlreiche Methoden entwickelt worden sind, diesem Problem zu begegnen. Hierzu gehören u.a. das Design Thinking, das Value Proposition Canvas, das damit verbundene Customer Development sowie das Lean-Startup-Konzept, das die Validierung von Minimal Viable Products (frühphasige Produktversionen mit Minimalfunktion)

\section{Product/Market Fit}

Der Product/Market Fit ist erreicht, wenn eine neue Lösung über die Zeit hinweg in den Zielmarkt so eingepasst wurde, dass sie aus Kundensicht nicht nur erkannt, sondern in großem und steigendem Umfang adaptiert wird. Hinzukommt für den Fit, dass mit der breiten Akzeptanz im Markt die Lösung auch für das innovierende Unternehmen rentabel wird. Der Fit beinhaltet auch, dass sich die Entwicklung von Kundenbasis und Rentabilität auf einem stabilen Niveau befinden. in den Vordergrund stellt. Allen Ansätzen ist gemein, mit der Marktvalidierung einer neuen Geschäftsidee bzw. Geschäftskonzeption so früh wie möglich anzusetzen und aussagekräftiges Feedback einzuholen.

Während die Notwendigkeit, derartiges Feedback zu erhalten, weitgehend unstrittig ist, kann für Startup-, aber auch CorporateEntrepreneure der damit verbundene Aufwand beträchtlich sein. Allein schon mit Blick auf das zeitliche Investment muss eine Vielzahl von Menschen unabhängig voneinander kontaktiert und Feedback intensiv

aufgearbeitet werden. Der Wunsch der Entrepreneure ist daher groß, die Einholung marktlicher Rückkopplungen zu bündeln, um trotz limitierter Ressourcen aussagekräftige, valide Erkenntnisse für die weitere Geschäftsmodellierung zu bekommen.

Dies wirft die Frage auf, wie diese Anforderungen konzeptionell umgesetzt werden können. Noch grundsätzlicher ist hingegen die Frage, wie Feedback auf eine unverfälschte und konstruktive Weise eingeholt werden kann, ohne den Fokus auf den Kern des Geschäftskonzepts zu verlieren. Warum ist das so schwierig?

- Entrepreneure glauben an sich und ihre Konzeption. Entsprechend tendieren sie manchmal dazu zu hören, was sie hören wollen. In der Forschung spricht man in solchen Fällen auch vom „Self-Serving Bias“, der dazu führt, dass Rückmeldungen schöner geredet werden als sie gemeint sind ${ }^{4}$.

- Zuweilen formulieren Entrepreneure auch dementsprechend ihre Fragen. Manchmal fragen sie so, dass die Antwort fast schon automatisch auf ihre Annahmen einzahlt, aber dann auch nicht mehr als die "halbe Wahrheit“ ist. Fragen, die auf den vermeintlich wunden Punkt der eigenen Geschäftskonzeption zielen, stellen sich rein psychologisch nicht leicht. 
- Auch die Feedback-Geber tun sich schwer, in einem grundsätzlich freundlichen Gespräch einem engagierten Menschen gegenüber eine ggfs. negative Rückkopplung in aller gebotenen Offenheit zu äußern. Kritik wird verpackt bzw. versteckt.

Vor diesem Hintergrund wird deutlich, dass sowohl das Setting als auch die beteiligten Personen auf die Einholung von Feedback auf dem Markt vorbereitet werden müssen. Ausgehend von dem Lösungsgedanken des Innovations-Quartiers steht damit die Beantwortung folgender Fragen im Mittelpunkt des vorliegenden Beitrags: (1) Was ist ein Inno-Quarter? (2) Wie gestaltet man Inno-Quarters zum Zwecke der Einholung validen Feedbacks vom Zielmarkt strukturell? (3) Welchen Nutzen bieten Inno-Quarters? Die Folgeschritte widmen sich dezidiert der Beantwortung und basieren auf eigener Feldarbeit der Autoren im Rahmen des von der EU im Interreg-Programm geförderten Projekts „Inno-Quarter". Die hier gespiegelten Erkenntnisse wurden über einem Zeitraum von drei Jahren im Rahmen von Feldbeobachtungen und Tiefeninterviews mit teilnehmenden Startup-Entrepreneuren, Organisatoren von Festivals und Living-Labs sowie mit im Projekt involvierten Business Coaches erhoben und im Rahmen des Interreg-EU-Projekts wissenschaftlich ausgewertet und diskutiert.

Im Rahmen der weiteren Vorgehensweise werden Inno-Quarters als Testbühnen für frühe und mittelreife Geschäftskonzepte verstanden, die in einer entspannten Atmosphäre Startup- und Corporate-Entrepreneuren Feedback von der Kundenseite ermöglichen (Living-Lab-Charakter). Inno-Quarters werden von Veranstaltern organisiert, welche Entrepreneure auf die Einholung von Feedback vorbereiten und die Einarbeitung von Feedback in die Geschäftskonzeption begleiten. Spezifischer Erfahrungshintergrund dieses Beitrags sind Festivals, die aufgrund der vorherrschenden Atmosphäre gute Voraussetzungen zur Einholung von Feedback bieten.

Nachfolgend werden Inno-Quarters zunächst aus der Perspektive der Events betrachtet, in die sie eingebettet sind. Sodann erfolgt ein Blick hinter die Kulissen, der erkennen lässt, wann und warum Inno-Quarters erfolgreich sind bzw. sein können. Dabei wird der Blick auf den gesamten Prozess gerichtet, der weit über das Event hinausreicht und im wahrsten Sinne des Wortes - schnell ein „100-Tage-Rennen“ der Geschäftsentwicklung 5 . Als Ausblick wird vorgestellt, wie Inno-Quarters auch außerhalb dieser Festivalund Event-Welt eine wirksame Bühne der Geschäftsentwicklung von Entrepreneuren darstellen können.

\section{Inno-Quarters auf Events - wie funktionieren sie und was steckt dahinter?}

Innovation kann kostspielig sein. Hingegen Festivals als Testgelände zu nutzen, gibt Entrepreneuren die Möglichkeit, ein großes, diverses und unvoreingenommenes Publikum ressourcenschonend zu erreichen. Allerdings unterscheiden sich Events erheblich u.a. in Sachen Infrastruktur und Publikum. Auch passt nicht jede Idee zu jedem Event. Daher ist das Inno-Quarter auf die zu testenden Lösungen anzupassen und das passende Event 
für die zu testenden Ideen auszuwählen, um ein Ideen/Event-Fit zu erreichen. Dies kann die Qualität des Feedbacks maßgeblich beeinflussen und den Erfolg der Idee beschleunigen. Man beachte: Festivals werden oft um (Sub-)Kulturen herum organisiert, was deren enorme Vielfalt erklärt, die von ländlichen bis zu städtischen Schauplätzen, unterschiedlichsten Themen sowie verschiedensten sozio-kulturellen Verankerungen reicht. Die Vielfalt kann eine Chance sein, aber auch das Risiko in sich bergen, beim Inno-Quarter nicht (nur) die Zielgruppe anzutreffen, sondern weit darüber hinausgreifende Gesellschaftsteile, was bei der Einholung und Auswertung von Feedback zu berücksichtigen ist. Im Interreg finanzierten EU-Projekt haben wir bisher mit großen Open AirKultur- und Musikfestivals (bis zu 200.000 Besucher) und Startup-Events (ca. 3.000 Besucher) kooperiert und dabei positive Erfahrung bezüglich der Durchführung von InnoQuarters gemacht. Um ein Inno-Quarter erfolgreich auf einem Festival zu etablieren, müssen sowohl der Inno-Quarter- als auch der Event-Host eng in Sachen Gestaltung, Aufbau und Logistik zusammenarbeiten, um die Wirksamkeit sicherzustellen. Darüber hinaus ist es nützlich, für die zu testenden Ideen relevante Praxispartner und potenzielle Kunden gezielt einzuladen.

\section{Standansatz vs. Integrierter Ansatz}

Inno-Quarters ermöglichen zwei unterschiedliche Ansätze zum Testen auf Festivals. Bei einem Stand-Ansatz werden Innovationsquartiere in Form von Pop-Up-Ständen auf dem Event eingerichtet. Jedes Startup bekommt einen eigenen Stand/Showcase, an dem es sich und seine Innovation präsentieren kann, um in Anschluss Feedback von Eventbesuchern einzuholen. Je nach Ziel des Entrepreneurs, können die Besucher an den Ständen auf unterschiedliche Art und Weise einbezogen werden - etwa durch Umfragen, Interviews, Beobachtungen, Produkttests und Feedbackkarten, aber auch mittels Prototypen oder kleinerer Experimente sowie Co-Creation-Aktivitäten, bei denen der Besucher selbst aktiv wird ${ }^{6}$.

Besonders auf Festivals können hohe Geräuschpegel auftreten. Hier ist es wichtig, die Stände in einer Umgebung abseits des Trubels zu platzieren. So können sich die Besucher zurückziehen und in einer entspannten Atmosphäre mit den Entrepreneuren in ein Gespräch treten. Eine naheliegende Möglichkeit, Aufmerksamkeit zu erregen und Besucher zum Living Lab anzulocken, sind die Bereitstellung einer Bühne und thematisch passende Programmpunkte, die eine spezielle Inno-Quarter Experience ermöglichen z.B. durch Pitch Events oder Expert Talks, die jedoch nicht von den eigentlichen Feedbackständen ablenken dürfen.

Doch wie kann man die Besucher dazu animieren, mit den Startups in einen Austausch zu treten? Während die Pop-Up-Stände von sich aus je nach Gestaltung Aufmerksamkeit erzeugen können, hat sich eine aktive Ansprache seitens des Entrepreneurs bewährt. Gleichwohl hat es sich als ausgesprochen nützlich erwiesen, für Eventbesucher und Feedbackgeber einen auf das Inno-Quarter bezogenen Anreizmechanismus zu 
implementieren, um in der Vielzahl der Reize eines Festivals Akzente setzen zu können. Über die Zeit bewährt hat sich ein ansprechendes Gesamtkonzept mit „gamifizierten“ Validierungsmethoden. So wurden im Rahmen des Inno-Quarter-Projektes FeedbackCoins als Belohnung für abgeschlossene Produkttest und Feedbackgespräche vergeben. An jedem Pop-Up-Stand konnten Besucher die Coins nach gegebenem Feedback sammeln und anschließend an dem Informationsstand des Inno-Quarters gegen attraktive, mit dem Quartier verbundene Prämien eintauschen.

\section{Beispiel: Klimakarl}

Das B2B-Startup Klimakarl bietet Unternehmen die Möglichkeit für interne Nachhaltigkeits-Challenges an. Mitarbeiter bilden Teams und treten im Büroalltag gegeneinander an, um möglichst viele Klimapunkte zu sammeln. Am Pop-Up-Stand auf dem Open Air-Kultur- und Musikfestival „Breminale“ in Bremen stellte Klimakarl die Idee in Form eines Posters dar und führte im Anschluss Interviews und Umfragen mit den Besuchern bezüglich ihrer App durch. Durch den Austausch mit den Festivalbesuchern wurden sie mit der Erkenntnis überrascht, dass eine klare Mehrheit der Teilnehmenden bereit war, ihre privaten Handys für eine Firmen-App zu nutzen. Anders als angenommen, waren die Datenschutzbedenken bei den Teilnehmern weitaus geringer. Diese Erkenntnis bildet das Fundament für Klimakarl, die jeweiligen Features der App und den korrespondierenden Entwicklungsprozess klarer zu priorisieren.

Im Gegensatz zum Standansatz verfolgt der integrierte Ansatz (Embeddded Approach) eine andere Strategie. Der eigentliche Quartiersgedanke auf dem Festival wird dadurch ersetzt, dass das gesamte Festival-Areal zur Testfläche für neue Lösungen bzw. Prototypen wird. Das bedeutet: die Entrepreneure mischen sich unter die Festivalgäste und testen ihre Ideen. Der Embedded Approach ist insbesondere vorteilhaft bei fortgeschritteneren Ideen, bei denen die Lösung bereits in Aktion getestet werden kann. Das Event bietet einen sicheren und überschaubaren Rahmen, in der die Performance erprobt sowie die User Experience und Akzeptanz des Produktes beobachtet werden können.

\section{Erfahrung zur Interaktion}

Aus den bisherigen Festivals konnten überwiegend positive Erfahrungen hinsichtlich der Interaktion zwischen Startups und Besuchern gemacht werden. Die Besucher waren offen und neugierig, sodass sich schnell Menschen um die Stände gesammelt haben. Insbesondere die Festival-Umgebung trägt zu einem ausgeprägten Gemeinschaftsgefühl bei, weil sie die Menschen auf eine recht ungezwungene Art zusammenbringt. Durch die Festivalatmosphäre entstanden angeregte Diskussionen, welche den Gründenden halfen, sich über die eigentlichen Tests hinaus zu inspirieren, neue Ideen auszutauschen und kreative Einblicke zu bekommen. Eine Herausforderung stellt die Tatsache dar, dass sich neben der Zielgruppe von Feedback-Gebenden auch andere Gäste für die InnoQuarters, die Stände und die Anreizmechanismen interessieren. Sie beleben die Szenerie, sind aber im Feedback-Prozess gesondert zu behandeln. 


\section{Beispiel: Eventbox by Worldperfect (DK)}

Das dänische Beratungsunternehmen Worldperfect, welches auf nachhaltige Event-Beratung spezialisiert ist, entwickelte ein Produkt für die nachhaltige Gestaltung von Veranstaltungen: die Eventbox - ein Abfallsortiersystem, welches die Besucher zu einem aktiven Teil der Lösung macht. Ziel von Eventbox ist es, Mülltrennung zu einem selbstverständlichen Teil jeder Veranstaltung zu machen und das Bewusstsein für Recycling sowohl bei Besuchern als auch bei Veranstaltern zu schärfen. Durch verschiedene Prototyping-Tests auf Events konnte das Unternehmen prüfen, inwieweit der Prototyp in der Praxis von Veranstaltern und Gästen angenommen wurde. Nach dem Event wurden einige Anpassungen an Konzept und Design durchgeführt. Hier stellte sich u.a. heraus, dass ein leichteres Material der Eventboxen vorteilhafter gewesen wäre, um die Handhabung für Event-Organisatoren zu vereinfachen. Neben dem Produkt selbst wurde außerdem getestet, wie sie Festivalbesucher des NorthSide-Festivals in Aarhus (Dänemark) dazu bringen können, Mülltrennung als begrüßenswerten, selbstverständlichen Teil des Alltags zu sehen. So nutzten sie anstelle des Wortes „Müll“, welches mit weniger attraktiven Dingen assoziiert wird, Wörter wie Ressource. Eine zentrale Erkenntnis war, dass es innen durch das Spiel mit Worten gelang, auf unterhaltsame und zugängliche Art das Thema mit positiven Assoziationen zu belegen. Zudem stellten sie ein Team zusammen, welches mit den Festivalbesuchern in Kontakt trat und Hilfestellung gab bei der Nutzung der Eventbox. Dem Team wurde der Name „Trash Talkers“ gegeben, um auf smarte Weise eine Gruppenidentität zu etablieren, die zugleich die Ansprache der Zielkunden unterstützt. Das Team „Trash Talkers“ fand sehr schnell Anklang bei den Festivalbesuchern und wurde ein großer Erfolg.

\section{Inno-Quarters als Lernprozess für Entrepreneure}

Verzerrtes Feedback und Rückmeldungen jenseits der Kernadressaten kann den Effekt dieses Instrument zum frühphasigen Testen von Innovationen und Produktideen auf Veranstaltungen konterkarieren. Deswegen sind eine sorgfältige und fokussierte Vorund Nachbereitung unumgänglich. Sie führen in einen ganzheitlichen prozessualen Ansatz mit folgenden Phasen (s. Abb. 1) und einem Zeitraum von ca. 100 Tagen (manchmal auch weniger): (1) Screening geeigneter Startups, (2) Matching der Startups mit geeigneten Events, (3) Auftakt-Workshop für teilnehmende Startups, (4) Entwicklung des Validierungskonzepts durch individuelle Coaching Sessions, (5) Validierung auf dem Event und (6) Begleitung der Implikationen für das Geschäftsmodell. 


\begin{tabular}{|l|ll}
\hline Ideen-Screening & Matching mit Event \\
• & Gesellschaftlicher Mehrwert & - Thematischer Schwerpunkt \\
- Testbarkeit & - Zielgruppen \\
\hline - Event Fit & - Vertragliche Rahmenbedingungen \\
\hline
\end{tabular}

\begin{tabular}{|c|c|c|}
\hline$\frac{5}{\frac{5}{20}}$ & $\begin{array}{l}\text { Auftakt-Workshop } \\
\text { für alle teilnehmenden Startups } \\
\text { - Methodische Grundlagen } \\
\text { - Erfahrungsberichte } \\
\text { - Team Building innerhalb der Kohorte }\end{array}$ & $\begin{array}{l}\text { Validierungskonzeption } \\
\text { durch individuelle Coaching Sessions } \\
\text { - Was wird getestet? } \\
\text { - Wie wird getestet? } \\
\text { - Was wird dokumentiert? }\end{array}$ \\
\hline
\end{tabular}

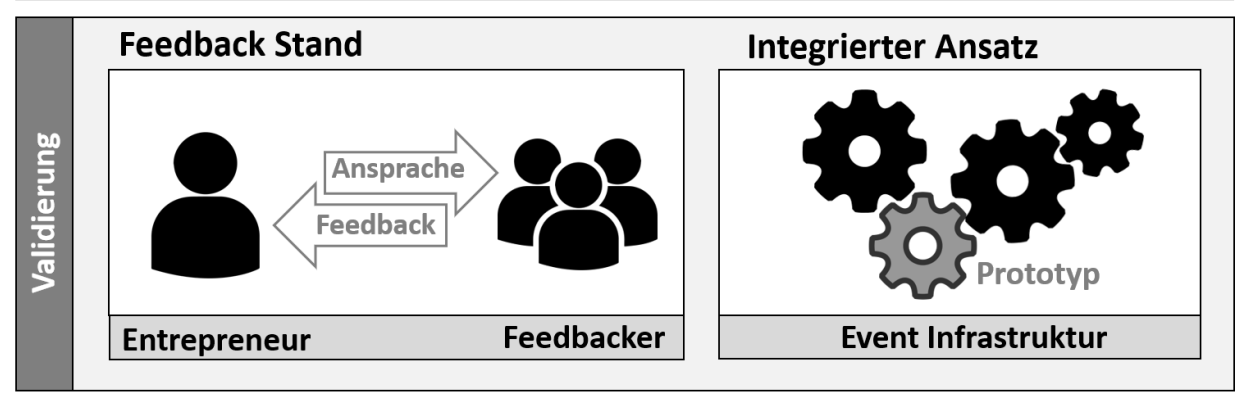

\begin{tabular}{|c|c|}
\hline $\begin{array}{l}\frac{\sum^{2}}{3} \\
\frac{3}{10} \\
\frac{0}{0} \\
\frac{0}{0} \\
\frac{0}{2}\end{array}$ & $\begin{array}{l}\text { Analyse der Implikationen für Geschäftsmodell } \\
\text { durch individuelle Coaching Sessions } \\
\text { - Rekapitulation der Eventtage } \\
\text { - Kategorisierung der Ergebnisse } \\
\text { - Ableitung der Kernerkenntnisse } \\
\text { - Maßnahmen zur Anpassung des Geschäftsmodells }\end{array}$ \\
\hline
\end{tabular}

Abbildung 1: Der Inno-Quarter-Prozess

\section{Phase 1: Screening geeigneter Startups}

Da im Rahmen des Programms mehrere Startup-Ideen und -Konzepte parallel getestet werden können, ist das Programm besonders wirkungsvoll, wenn es sich pro Event auf definierte Themen fokussiert. So können die teilnehmenden Entrepreneure durch die Einbindung themenspezifischer Partner wirkungsvoll vorbereitet werden. Auch mit Blick auf Veranstaltungsbesucher kann so dazu beigetragen werden, Interesse und Verständnis des Inno-Quarter-Konzepts zu erhöhen. Daher bietet es sich an, geeignete Auswahlkriterien zu definieren, die eine Idee auszeichnen sollte, um im Rahmen des Programms berücksichtigt werden zu können. Das EU-Projekt Inno-Quarter unterstützt so beispielsweise Ideen, die einen starken Nachhaltigkeitsfokus haben. Es setzt ferner sog. Innovation Brokers ein, die anhand definierter Kriterien den Fit bestimmen und bei der 
Selektionsentscheidung mittels ihrer eingebrachten Expertise behilflich sind. Weiterhin wird bei der Suche nach Startups auch auf den Ideen/Event-Fit geachtet sowie das Vorhandensein einer innovativen testbaren Idee. Der Ideen/Event-Fit beschreibt das Zusammenpassen der zu testenden Idee mit der Veranstaltung, auf der die Produktvalidierung durchgeführt werden soll (siehe Phase 2). Das Kriterium der testbaren Idee beschreibt, dass der geplante Test räumlich, zeitlich und mit Blick auf vorhandene lokale Gegebenheiten durchgeführt werden kann. Kernaufgabe im Screening ist, durchgehend nach frühphasigen Startups zu suchen, die ihre Idee am Markt testen wollen. Aufgrund entsprechender Dichte an geeigneten Projekten haben sich für das Screening v.a. folgende Formate bewährt: Pitch-Events und Ideenwettbewerbe, Akzelerator- oder Inkubatorprogramme sowie das Screening in Entrepreneurship-Kursen von Universitäten. Neben dem Screening bietet Inno-Quarter auch die Möglichkeit der Selbstbewerbung von Startups über die Website www.innoquarter.eu. Die Teilnahme am Inno-QuarterProgramm ist für Startups aufgrund der EU-Förderung unentgeltlich.

\section{Phase 2: Matching der Startups zu Inno-Quartern/Events}

Im nächsten Schritt geht es um das Matching der ausgewählten Startups mit geeigneten Veranstaltungen. Wie beschrieben, ist eines der essentiellen Auswahlkriterien der Ideen/Event-Fit, da jede Veranstaltung andere Arten von Gästen anzieht und entsprechend unterschiedliche Zielgruppen auch für die zu testenden Ideen vorweisen kann. So lassen sich beispielsweise auf Musikfestivals aufgrund der Besucher der Veranstaltungen insbesondere Lebensmittel- und B2C-Ideen mit einer sehr breiten Zielgruppe testen. B2B-Ideen hingegen lassen sich auf Messeveranstaltungen mit Fachpublikum besser in Szene setzen. Auch sind die Bedingungen der Veranstalter der Events vorab zu klären, da etwa Verträge mit Cateringfirmen oder Brauereien bestehen können, die den exklusiven Vertrieb von Nahrungsmitteln auf der Veranstaltung regeln und entsprechend Produkte in dieser Kategorie daher für Tests ggfs. ausschließen. Oft finden Veranstaltungen auch unter einem definierten Motto statt. Idealerweise können dann auch thematisch passende Ideen getestet werden, um Veranstaltungsbesuchern ein kohärentes Bild zu liefern. Abhängig von der Größe der Veranstaltung wurden im EU-Projekt pro Event zwischen 5 und 10 Ideentests durchgeführt, um die zur Verfügung gestellte Standfläche auf den Veranstaltungen sinnvoll zu nutzen. Festivals und Events sind erfahrungsgemäß motiviert, Inno-Quarters als Teil des Festivals aufzunehmen, da die innovativen und nachhaltigen Startups den Veranstaltern Ideen und Konzepte für nachhaltiges Wirtschaften liefern. Gerade mit Blick auf das hohe Müllaufkommen auf Festivals, aber auch die Strom- und Wasserversorgung auf solchen Events, sind Fragestellungen, mit denen sich die Eventveranstalter immer wieder beschäftigen. Auch sind innovative und nachhaltige Themen oft im Einklang mit den Interessen der Zielgruppen der Veranstaltung. Daher stellt Inno-Quarter auch aus Veranstaltersicht einen abwechslungsreichen Unterhaltungsaspekt dar, sodass Eventveranstalter sehr zugänglich dafür sind, Innovationsquartiere auf dem Festivalgelände zu integrieren. 


\section{Phase 3: Auftaktveranstaltung}

Sind geeignete Startups für eine Veranstaltung fixiert, beginnt das „On-Boarding“. Es hat sich als sinnvoll erwiesen, eine Auftaktveranstaltung für alle involvierten Entrepreneure zu organisieren. Auf diese Weise können zunächst die für alle geltenden Rahmenbedingungen und Programmabfolgen vorgestellt und Fragen geklärt werden. Hier werden insbesondere Kernüberlegungen des Programms vermittelt. Dazu gehört die Vermittlung der Wichtigkeit frühphasiger Tests und der Herausforderungen bei der Durchführung. Auch können an dieser Stelle die "Go's" und „No-Go's" als Erfahrungsberichte vorheriger Kohorten geteilt werden. Zur Auftaktveranstaltung können zudem gezielt Fachexpert:innen passend zur jeweiligen Kohorte eingeladen werden, die Tipps und Kontakte vermitteln können. Zum Abschluss erhalten die Startups zudem gezielte Fragestellungen für die Vorbereitung ihrer Produktvalidierung (relevant für Phase 4). Besonders wichtig ist eine gemeinsame Auftaktveranstaltung v.a. auch zur Schaffung eines Gemeinschaftsgefühls. Während der Auftaktveranstaltung stellen alle Entrepreneure ihre Idee vor der gesamten Kohorte vor und lernen sich in gezielt geplanten Netzwerkpausen besser kennen, damit eine Grundlage für einen vertrauensvollen gegenseitigen Austausch geschaffen werden kann. Es hat sich gezeigt, dass eben dieser Erfahrungsaustausch zwischen den Teilnehmenden vor allem im späteren Verlauf des Programms einen großen Mehrwert für die Entrepreneure hat, da diese sich alle in ähnlich frühen Phasen befinden und auf diese Weise ihre Learnings wechselseitig austauschen und reflektieren können.

\section{Phase 4: Individuelle Vorbereitung}

Da jedes Startup ganz eigene produkt- oder prozessbezogene Problemstellungen im Rahmen des Programms validieren möchte, ist ein individuelles Coaching mit erfahrenen Business Coaches und den einzelnen Entrepreneuren oder Startup-Teams notwendig. In der individuellen Vorbereitung wird zunächst diskutiert, welche exakte Fragestellung im Rahmen des Festivals getestet werden soll. Im nächsten Schritt geht es um die Diskussion und Auswahl geeigneter möglicher Testarten und die Spezifizierung der Testabfolge. Hier bietet es sich an, einen Prototypen oder ein Minimal Viable Product der Idee in den Test einzubinden, um das Verständnis der Festivalbesucher zu erhöhen und somit die Interaktion zwischen Feedbackgeber und Entrepreneur zu erleichtern. Ausschließlich Gespräche über eine Idee ohne jegliche Artefakte dieser Art zu führen, z.B. um zunächst die Zielgruppe besser zu verstehen, sind grundsätzlich auch möglich, sind aber nicht selten abstrakter. Feedbackgespräche waren bisher zielführender, wenn die Idee visuell und haptisch veranschaulicht werden konnte. Die individuelle Vorbereitung umfasst zudem auch die Schulung der Entrepreneure, wie Fragen in Feedbackgesprächen offen und ungelenkt gestellt werden können, um die Antworten der Feedbackgeber nicht zu beeinflussen. Auch wird auf den in der Wissenschaft diskutierten Confirmation Bias $^{7}$ aufmerksam gemacht. Er beschreibt, dass Menschen bei der Suche nach 
Informationen dazu neigen, solchen Informationen mehr Aufmerksamkeit zu schenken, die ihren eigenen Überzeugungen und Meinungen entsprechen und gegenteilige Meinungen tendenziell vernachlässigen. Um diesem Verzerrungseffekt der eigenen Wahrnehmung während der Produkttests vorzubeugen, wird das systematische Festhalten von Feedback als abschließender Punkt der Vorbereitung gemeinsam entwickelt. Die systematische Erfassung des einzuholenden Feedbacks ist für die spätere Auswertung essentiell, da auf Festivals mit einem hohen Besucherstrom zu rechnen ist und damit das Eintreten des Confirmation Bias nach mehreren geführten Gesprächen mit positiven Rückmeldungen zur Idee leicht aufkommen kann und die kritischen Anmerkungen ggfs. untergehen könnten.

\section{Phase 5: Validierung auf Events}

Der Kern des Inno-Quarter-Ansatzes ist das Testen der Idee auf Festivalveranstaltungen. Je nach Auswahl des Testansatzes ist der Aufbau des Tests rechtzeitig zu planen. Bei dem Ansatz „Pop-Up-Stand“ ist der Aufbau des Standes am Tag vor dem Event in der Regel ausreichend. Wurde ein Embedded-Ansatz mit Integration des Tests in der Infrastruktur des Festivals geplant, so ist hier entsprechend frühzeitiger mit den Festivalorganisatoren die Vorbereitung anzugehen. Für die Festival-Tage haben sich aus Entrepreneur-Sicht folgende Aspekte als nützlich erwiesen:

- personelle Unterstützung aus dem eigenen Team oder Freundeskreis zur Bewältigung der vielen, nicht selten ungeplanten Aufgaben im Kontext der Feedbackgespräche - auch um Pausen zu ermöglichen,

- striktes Einhalten der in der Vorbereitung besprochenen Abläufe,

- systematisches Festhalten des Feedbacks.

Es kommt durchaus vor, dass während der Veranstaltung z.B. Testinhalte nicht wie geplant funktionieren oder Tests auf eine andere Weise noch zielgerichteter durchgeführt werden können, sodass spontan Anpassungen möglich sind. Um durch eine Umgestaltung des Tests die Testergebnisse aber nicht zu verfälschen, ist hier eine Rücksprache mit den Business Coaches empfehlenswert, die aus diesem Grund ebenfalls vor Ort sein sollten.

Die Festivalbesucher müssen vorab im Übrigen nicht im Vorhinein auf die Tests vorbereitet werden. Zur Kennzeichnung des Inno-Quarters wurden Erklärschilder bzgl. des Konzepts aufgestellt und das Inno-Quarter-Team hat zuweilen das Laufpublikum angesprochen und auf die Feedbackstände aufmerksam gemacht. Durchschnittlich haben Feedbackgespräche im Rahmen der Veranstaltung ca. 15-20 Minuten gedauert und je nach Länge der Veranstaltung und Anzahl an Standbetreuern wurden zwischen 40 und 60 Feedbackgespräche pro Startup und Event durchgeführt. Da die Fragestellungen der Startups sehr unterschiedlich waren - von Designaspekten über Geschäftsmodelle und 
Produktspezifikationen -, ist weniger die Quantität der Gespräche entscheidend als die Intensität der Validierungen.

Welche Gäste tatsächlich zur potentiellen Zielgruppe der Startups gehörten und damit von besonderer Relevanz für die Feedbackgespräche sind, wurde mit gezielten Fragestellungen zu Beginn der Validierungen zu ermitteln versucht, die im Vorbereitungscoaching geübt wurden.

\section{Phase 6: Begleitung der Implikationen für das Geschäftsmodell}

Die individuelle Nachbereitung der Entrepreneure sollte möglichst direkt nach dem Festival angesetzt werden, da die Eindrücke und Erfahrungen der Validierungsgespräche noch frisch sind, aber schnell in Vergessenheit geraten können. Bei der Nachbereitung setzen sich die Entrepreneure individuell mit dem Business Coach zusammen und gehen die systematisch erfassten Erkenntnisse der Feedbackgespräche durch. Hier ist es notwendig, zunächst beschreibend das Feedback wiederzugeben, um sich einen Überblick über Inhalte und Häufung von Themen zu verschaffen. Der Entrepreneur sollte die systematisch erfassten Daten dabei möglichst geeignet kategorisieren und mit der eigenen Erfahrung reflektieren. Kundenmeinung ist wichtig, gleichzeitig ist jedes Feedbackgespräch eine Individualmeinung und muss in den Gesamtkontext eingeordnet werden. Im Anschluss definiert jeder Entrepreneur seine Key Learnings sowie die daraus abzuleitenden Konsequenzen und Maßnahmen für die Weiterentwicklung des eigenen Konzepts. Aufgabe des Business Coaches ist es, diesen Prozess kritisch zu begleiten und bei Bedarf beratend zu intervenieren. Je nach Learnings kann dann die Weiterentwicklung des Konzepts abgebrochen, fortgesetzt oder eine weitere Feedbackschleife durch das erneute Teilnehmen an Inno-Quarter auf einem anderen Festival angegangen werden.

Ist keine weitere Testung auf einem weiteren Inno-Quarter Festival geplant, so endet mit der Nachbereitung der formale Zyklus des Inno-Quarter-Konzeptes. Informelle Alumni-Veranstaltungen oder das Einrichten geteilter Kommunikationskanäle wie Chatgruppen fördern über das Programmende hinaus den Erfahrungsaustausch der teilnehmenden Entrepreneure.

\section{Inno-Quarters - vom Event zur allgemeinen Innovationsbühne}

Inno-Quarters wurden in diesem Beitrag in den Kontext der Events, speziell in den Festival-Kontext gestellt. Als Living-Labs zur Feedbackeinholung und einem damit verbundenen Co-Development trägt der zentrale Gedanke dieses Formats aber auch weit über diesen Kontext hinaus. Während mit Festivals in erster Linie B2C-Konstellationen erfasst werden können, wird der B2B- und B2A-Kontext nicht zuletzt über Inno-Quarter auf Messen und Ausstellungen bespielbar. Wie bei Festivals ist auch hier eine recht kostengünstige und zugleich wirkungsvolle Feedback-Einholung für neue Geschäftskonzepte möglich, wenn die Quartiere im Sinne der o.g. Prinzipien betrieben werden. Auch wenn der Inno-Quarter-Prozess über mehrere Monate geht, was Vorbereitung, Durchführung 
und Nachbereitung anbelangt, so ist diese Form des Feedback-basierten Co-Developments dennoch sehr zeitsparend aus Sicht der Entrepreneure.

Vergegenwärtigt man sich die Wirkungen von Inno-Quarters, so lassen sie sich aber auch in anderen Kontexten erzielen. Während die Ansiedlung in klassischen Marktveranstaltungen noch recht intuitiv und in der Umsetzung zum oben Gesagten durchaus ähnlich ist, ändert sich das Bild, wenn man sie in die Szenerie des Business Developments von Innovations- bzw. Gründungsökosystemen einbringt. Hier könnten Inkubatoren und Akzeleratoren einen passenden und permanent verfügbaren Hintergrund liefern. Sie würden vor allem dann zum Tragen kommen, wenn innerhalb der Inkubationsund Akzelerationsräume Events angesetzt werden, die neben den Stamm-Akteuren auch „Laufgäste“ einbeziehen. Ähnliches gilt für Formate des Corporate Entrepreneurship, in denen Inno-Quarters entweder temporär oder sogar dauerhaft angesiedelt werden.

Nicht zuletzt der Übergang in die pandemische Zeit hat auch erkennen lassen, dass der Grundgedanke des Inno-Quarters von einer physischen Umgebung entkoppelt werden kann. Ein virtuelles Event zur Testung von Prototypen hat etwa gezeigt, dass sich die Wirkungen im virtuellen Raum fast in gleicher Weise wie in physischen Räumen einstellen. Diese Erfahrungen verstärken den Eindruck, dass Inno-Quarters als Vehikel zur Verstärkung explorativer Vorstöße von Entrepreneuren und etablierten Unternehmen von grundlegender Bedeutung sein und eine entsprechende Verankerung finden können.

\section{Förderungserklärung}

Forschung, Autorenschaft und Entwicklung des vorliegenden Beitrags entstand im Rahmen des Projekts „Inno-Quarter", das finanziell durch Interreg Northsea Region European Regional Development Fund gefördert wird.

\section{Literatur}

1 Vgl. hierzu Blank, S. (2013). Why the Lean Start-Up Changes Everything. Harvard Business Review, 91(5), 6372; Blank, S. (2020). The Four Steps to the Epiphany, 5. Aufl., Hoboken, NJ: Wiley; Ries, E. (2011). The Lean Startup, New York: Crown.

2 Vgl. Dijkstra, A.; Boonstra, M. (2021). Festival Experimentation Guide, Leeuwarden: NHL Stenden.

3 Vgl. CB Insights. (2018). Top 20 Reasons Why Startups Fail. Aufgerufen am 23. Mai 2019: https://www.cbinsights.com/research/startup-failure-reasons-top/

4 Vgl. Miller, D. T.; Ross, M. (1975). Self-serving biases in the attribution of causality: Fact or fiction? Psychological Bulletin, 82, 213-225.

5 Ähnlich: Freiling, J.; Holi, M.; Stührenberg, K. (2020). Exploration durch digitale Transformationsräume. Austrian Management Review, 10, 47-59.

6 Vgl. Osterwalder; A. (2020). Testing Business Ideas - A Field Guide for Rapid Experimentation. Hoboken/NJ: Wiley.

7 Vgl. York, J.L.; Danes, J.E. (2014). Customer development, innovation, and decision-making biases in the lean startup. Journal of Small Business Strategy, 24(2), 21-39. 


\section{Information zu den AutorInnen}

Prof. Dr. Jörg Freiling ist Inhaber des Lehrstuhls für Mittelstand, Existenzgründung und Entrepreneurship (LEMEX) an der Universität Bremen und Prodekan des Fachbereichs 7 (Wirtschaftswissenschaft). Seine Forschungsinteressen liegen in den Gebieten Transnational Entrepreneurship, Gründungsökosysteme, Entrepreneurial Support Systems, Corporate Entrepreneurship und Geschäftsmodellinnovation.

Dr. Thomas Baron ist wissenschaftlicher Mitarbeiter und Postdoc am Lehrstuhl für Mittelstand, Existenzgründung und Entrepreneurship (LEMEX) an der Universität Bremen. Seine Forschung fokussiert regionale Gründungsökosysteme, transnationales Unternehmertum und Gründungsunterstützungsprogramme.

Quynh Duong Phuong, M.Sc., ist wissenschaftliche Mitarbeiterin und Doktorandin am Lehrstuhl für Mittelstand, Existenzgründung und Entrepreneurship (LEMEX) an der Universität Bremen. Ihre Forschungsinteressen liegen im Bereich Entrepreneurship Education und Student Entrepreneurship.

Jan Elsner, M.Sc., ist Doktorand am Lehrstuhl für Mittelstand, Existenzgründung und Entrepreneurship (LEMEX) an der Universität Bremen mit aktuellen Forschungsfokus in den Bereichen ,entrepreneurial learning' und, event-based business validation'. Er ist zudem Gründer eines E-Health Startups im Gebiet der Dermatologie mit Standorten in Berlin und Bremen. 